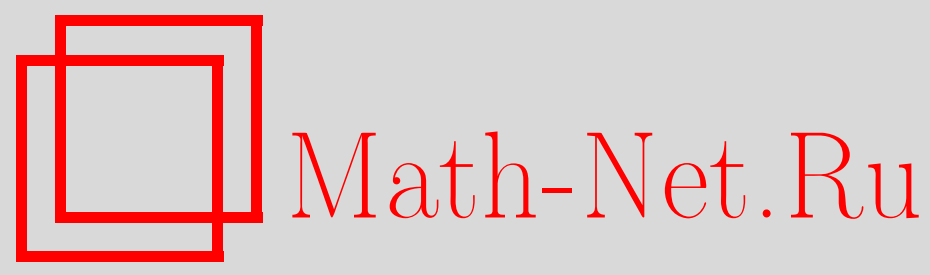

В. И. Буслаев, С. Ф. Буслаева, О композициях дробнолинейных преобразований, Матем. заметки, 1997, том 61, выпуск 3, 332-338

DOI: https://doi.org/10.4213/mzm1507

Использование Общероссийского математического портала Math-Net.Ru подразумевает, что вы прочитали и согласны с пользовательским соглашением http://www . mathnet.ru/rus/agreement

Параметры загрузки:

IP : 3.85 .5 .30

26 апреля 2023 г., 14:40:05

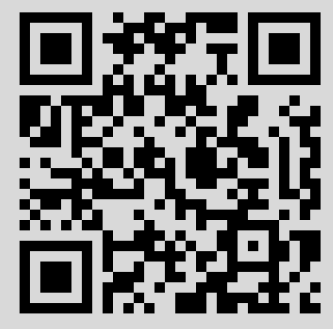




\title{
О КОМПОЗИЦИЯХ ДРОБНО-ЛИНЕЙНЫХ ПРЕОБРАЗОВАНИЙ
}

\author{
В.И. Буслаев, С.Ф. Буслаева
}

Статья посвящена вопросу предельного поведения композиций $\left(\mathbf{S}_{n} \circ \cdots \circ \mathbf{S}_{1}\right)(z)$ и $\left(\mathbf{S}_{1} \circ \cdots \circ \mathbf{S}_{n}\right)(z)$ дробно-линейных преобразований $\mathbf{S}_{n}(z)(n=1,2, \ldots)$, неподвижные точки которых имеют пределы. В частном случае, когда дробно-линейные преобразования имеют вид $\mathbf{S}_{n}(z)=\alpha_{n}\left(\beta_{n}+z\right)^{-1}$, последовательность композиций $\left(\mathbf{S}_{1} \circ \cdots \circ \mathbf{S}_{n}\right)(z)$ в точке $z=0$ совпадает с последовательностью подходящих дробей формальной непрерьвной дроби

$$
\frac{\alpha_{1}}{\beta_{1}+\frac{\alpha_{2}}{\beta_{2}+\cdots}} .
$$

В этой связи полученный результат находит применение и в вопросах сходимости формальных непрерьвных дробей.

Библиография: 6 названий.

Основное содержание данной статьи заключено в следующих теоремах.

ТЕОрема 1. Пусть неподвижные точки $p_{1, n}$ и $p_{2, n}$ последовательности невырохсденных дробно-линейных преобразований $\mathbf{S}_{n}(z)(n=1,2, \ldots)$ таковы, что

$$
\lim _{n \rightarrow \infty} p_{1, n}=p_{1}, \quad p_{2, n} \in K \subset \overline{\mathbb{C}} \backslash p_{1} \quad\left(n \geqslant n_{0}\right), \quad \underline{\lim }\left|\mathbf{S}_{n}^{\prime}\left(p_{1, n}\right)\right|>1,
$$

әде $K$ - некоторый компакт на сфере Римана, не содержащий $p_{1}$. Тогда при всех $z \in \overline{\mathbb{C}} \backslash p_{1}$ существует предел композииии $\left(\mathbf{S}_{1} \circ \cdots \circ \mathbf{S}_{n}\right)(z)$ при $n \rightarrow \infty, u$ этот предел не зависит от $z$.

Простой пример $\mathbf{S}_{n}(z)=2 z+(-2)^{-n}$ показывает, что в точке $p_{1}$ (в данном примере $\left.p_{1}=0\right)$ предела композищий $\left(\mathbf{S}_{1} \circ \cdots \circ \mathbf{S}_{n}\right)(z)$ действительно не существует.

Как показьвает следующая теорема, для композиций $\left(\mathbf{S}_{n} \circ \cdots \circ \mathbf{S}_{1}\right)(z)$ дробно-линейных преобразований имеет место в некотором смысле двойственное утверждение.

ТЕОрема 2. Пусть неподвижные точки $p_{1, n}$ и $p_{2, n}$ последовательности невьрожсденных дробно-линейных преобразований $\mathbf{S}_{n}(z)(n=1,2, \ldots)$ таковы, что

$$
\begin{gathered}
\lim _{n \rightarrow \infty} p_{2, n}=p_{2}, \\
p_{1, n} \in K \subset \overline{\mathbb{C}} \backslash p_{2} \quad\left(n \geqslant n_{0}\right), \\
\varlimsup_{n \rightarrow \infty}\left|\mathbf{S}_{n}^{\prime}\left(p_{2, n}\right)\right|<1,
\end{gathered}
$$

Работа выполнена при финансовой поддержке Российского фонда фундаментальных исследований, грант № 96-01-00659. 
где $K$ - некоторый компакт на сфере Римана, не содержащий $p_{2}$. Тогда при всех $z \in \overline{\mathbb{C}}$, за исключением некоторой точки $z_{0}$, существует предел композиции $\left(\mathbf{S}_{n} \circ \cdots \circ \mathbf{S}_{1}\right)(z)$ при $n \rightarrow \infty, u$ әтот предел равен $p_{2}$.

В дополнение к теореме 2 можно добавить, что если условие (2) заменить более сильным условием

$$
\lim _{n \rightarrow \infty} p_{1, n}=p_{1} \neq p_{2}
$$

то можно утверждать, что в исключительной точке $z_{0}$ также существует предел композиции $\left(\mathbf{S}_{n} \circ \cdots \circ \mathbf{S}_{1}\right)(z)$, и этот предел равен $p_{1}$.

Напомним, что неподвижная точка $p$ дробно-линейного преобразования $\mathbf{S}(z)$ назьвается “притягивающей", если $\left|\mathbf{S}^{\prime}(p)\right|<1$, “отталкивающей”, если $\left|\mathbf{S}^{\prime}(p)\right|>1$, и “нейтральной", если $\left|\mathbf{S}^{\prime}(p)\right|=1$. Обратим внимание, что если в условиях теоремы 1 говорится о наличии предела "отталкивающих" неподвижных точек, то в условиях теоремы 2 говорится о наличии предела "притягивающих" неподвижных точек". Легко проверяется, что для всякого дробно-линейного преобразования $\mathbf{S}(z)$ имеет место равенство $\mathbf{S}^{\prime}\left(p_{1}\right) \mathbf{S}^{\prime}\left(p_{2}\right)=1$, где $p_{1,2}-$ его неподвижные точки.

Как уже упоминалось и как более детально будет показано ниже, в применении к вопросам сходимости формальных непрерьвных дробей теорема 1 может быть переформулирована в следующем виде.

ТЕОРема 3. Пусть формальная непрерывная дробь

$$
\frac{\alpha_{1}}{\beta_{1}+\frac{\alpha_{2}}{\beta_{2}+\cdots}}
$$

такова, что

$$
\begin{gathered}
0<c_{1} \leqslant\left|\alpha_{n}\right| \leqslant c_{2}<\infty \quad\left(n \geqslant n_{0}\right), \\
\lim _{n \rightarrow \infty}\left(\alpha_{n}-p \beta_{n}\right)=p^{2},
\end{gathered}
$$

әде $c_{1}, c_{2}$ - некоторые положительные постоянные, $p$ - некоторая фиксированная точка комплексной плоскости с модулем, строго превосходящим $c_{2}^{1 / 2}$. Тогда эта непрерывная дробь сходится.

Простым следствием теоремы 3 является хорошо известная в теории непрерьвных дробей теорема Ван $\Phi$ лека [1].

ТЕОРемА ВАН ФЛЕКА. Пусть формальная непрерывная дробь (4) такова, что $\beta_{n}=1(n=1,2, \ldots)$,

$$
\lim _{n \rightarrow \infty} \alpha_{n}=\alpha, \quad \alpha \neq 0, \quad \alpha \notin\left[-\infty,-\frac{1}{4}\right] .
$$

Тогда эта непрерывная дробъ сходится.

Действительно, пусть выполняются предположения теоремы Ван Флека. Тогда неравенство (5) вьполнено при $n \geqslant n_{0}$ с постоянными $c_{1}, c_{2}$, как угодно близкими к $|\alpha|$. Так как $p_{1} p_{2}=-\alpha$, где $p_{1,2}-$ корни квадратного уравнения $\alpha-z-z^{2}=0$, и равенство 
$\left|p_{1}\right|=\left|p_{2}\right|$ возможно только лишь при $\alpha \in[-\infty,-1 / 4]$, то один из корней (пусть для определенности $\left.p_{1}\right)$ по модулю строго больше $|\alpha|^{1 / 2}$. При этом

$$
\lim _{n \rightarrow \infty}\left(\alpha_{n}-p_{1} \beta_{n}\right)=\alpha-p_{1}=p_{1}^{2} .
$$

Таким образом, условие (6) теоремы 3 также выполнено и, следовательно, непрерывная дробь по теореме 1 сходится.

Теоремы 1 и 2 будут получены как следствие соответствуюших утверждений о предельном поведении произведений линейных операторов в двумерном векторном пространстве.

ЛЕмма 1. Пусть в двумерном векторном пространстве имеется последовательность невырожсденных линейных операторов $\mathbf{A}_{n}(n=1,2, \ldots)$, задаваемых в некотором фиксированном базисе $\mathbf{e}_{1}, \mathbf{e}_{2}$ равенствами $\mathbf{A}_{n} \mathbf{e}_{1}=\alpha_{n} \mathbf{e}_{1}+\gamma_{n} \mathbf{e}_{2}, \mathbf{A}_{n} \mathbf{e}_{2}=$ $\beta_{n} \mathbf{e}_{1}+\delta_{n} \mathbf{e}_{2}$, zде $\alpha_{n} \neq 0\left(n \geqslant n_{0}\right)$,

$$
\lim _{n \rightarrow \infty} \gamma_{n} \alpha_{n}^{-1}=0, \quad \varlimsup_{n \rightarrow \infty}\left|\beta_{n} \alpha_{n}^{-1}\right|<\infty, \quad \varlimsup_{n \rightarrow \infty}\left|\delta_{n} \alpha_{n}^{-1}\right|<1 .
$$

Тогда существуют два линейно независимых вектора $\mathbf{f}_{1}$ и $\mathbf{f}_{2}$ таких, что

$$
\begin{aligned}
& \lim _{n \rightarrow \infty} D_{n}^{-1} \mathbf{A}_{n} \cdots \mathbf{A}_{1} \mathbf{f}_{1}=\mathbf{e}_{1}, \\
& \lim _{n \rightarrow \infty} D_{n}^{-1} \mathbf{A}_{n} \cdots \mathbf{A}_{1} \mathbf{f}_{2}=0,
\end{aligned}
$$

где $D_{n}=\left[\mathbf{A}_{n} \cdots \mathbf{A}_{1} \mathbf{f}_{1}\right]^{1}$ - первая координата вектора $\mathbf{A}_{n} \cdots \mathbf{A}_{1} \mathbf{f}_{1}$.

Отметим, что лемма 1 представляет собой усиленньй вариант классических теорем Пуанкаре [2] и Перрона [3] о рекуррентных соотношениях (см. также [4]). Действительно, векторная (более общая по сравнению с первоначальной) формулировка теорем Пуанкаре и Перрона (см. [5], [6]) накладьвает на последовательность операторов $\mathbf{A}_{n}(n=1,2, \ldots)$ более жесткие, по сравнению с леммой 1 , требования:

$$
\lim _{n \rightarrow \infty} \alpha_{n}=\alpha, \quad \lim _{n \rightarrow \infty} \beta_{n}=\lim _{n \rightarrow \infty} \gamma_{n}=0, \quad \lim _{n \rightarrow \infty} \delta_{n}=\delta, \quad|\alpha|>|\delta| .
$$

Отметим также, что утверждение, аналогичное лемме 1 , имеет место и в векторном пространстве произвольной размерности.

ДОКАЗАТЕЛЬСТВО ЛЕММЫ 1. Из условий леммы следует, что при всех $n \geqslant n_{1} \geqslant n_{0}$ $\left|\delta_{n} \alpha_{n}^{-1}\right| \leqslant 1-\delta(\delta>0),\left|\beta_{n} \alpha_{n}^{-1}\right| \leqslant c(c<\infty),\left|\gamma_{n} \alpha_{n}^{-1}\right| \leqslant \varepsilon$, где в качестве $\varepsilon$ фиксируем число $\varepsilon=\delta^{2}(9 c)^{-1}$. Будем обозначать через $u_{n}(\mathbf{f})$ и $v_{n}(\mathbf{f})$ первую и вторую координаты вектора $\mathbf{A}_{n} \cdots \mathbf{A}_{1} \mathbf{f}$ в базисе $\mathbf{e}_{1}, \mathbf{e}_{2}$. Если $\left|u_{n}(\mathbf{f}) / v_{n}(\mathbf{f})\right| \geqslant c^{1 / 2} \varepsilon^{-1 / 2}$, то

$$
\begin{aligned}
\left|\frac{u_{n+1}(\mathbf{f})}{v_{n+1}(\mathbf{f})}\right| & =\left|\frac{\alpha_{n+1} u_{n}(\mathbf{f})+\beta_{n+1} v_{n}(\mathbf{f})}{\gamma_{n+1} u_{n}(\mathbf{f})+\delta_{n+1} v_{n}(\mathbf{f})}\right| \geqslant \frac{\left|u_{n}(\mathbf{f})\right|-c\left|v_{n}(\mathbf{f})\right|}{\varepsilon\left|u_{n}(\mathbf{f})\right|+(1-\delta)\left|v_{n}(\mathbf{f})\right|} \\
& \geqslant \frac{1-c\left(\varepsilon^{1 / 2} c^{-1 / 2}\right)}{\varepsilon+(1-\delta)\left(\varepsilon^{1 / 2} c^{-1 / 2}\right)} \geqslant c^{1 / 2} \varepsilon^{-1 / 2} .
\end{aligned}
$$

Таким образом, если неравенство $\left|u_{n}(\mathbf{f}) / v_{n}(\mathbf{f})\right| \geqslant c^{1 / 2} \varepsilon^{-1 / 2}$ вьполнено при некотором $n_{2} \geqslant n_{1}$, то оно вьполнено при всех $n \geqslant n_{2}$. 
В качестве вектора $\mathbf{f}_{1}$ возьмем вектор $\mathbf{f}_{1}=\mathbf{A}_{1}^{-1} \cdots \mathbf{A}_{n_{1}}^{-1} \mathbf{e}_{1}$. Тогда $u_{n_{1}}\left(\mathbf{f}_{1}\right)=1$, $v_{n_{1}}\left(\mathbf{f}_{1}\right)=0$. По доказанному $\left|u_{n}\left(\mathbf{f}_{1}\right) / v_{n}\left(\mathbf{f}_{1}\right)\right| \geqslant c^{1 / 2} \varepsilon^{-1 / 2}$ при всех $n \geqslant n_{1}$ и, следовательно,

$$
\begin{aligned}
\left|u_{n}\left(\mathbf{f}_{1}\right)\right| & =\left|\alpha_{n} u_{n-1}\left(\mathbf{f}_{1}\right)+\beta_{n} v_{n-1}\left(\mathbf{f}_{1}\right)\right| \geqslant\left(1-c\left(\varepsilon^{1 / 2} c^{-1 / 2}\right)\right)\left|\alpha_{n}\right| \cdot\left|u_{n-1}\left(\mathbf{f}_{1}\right)\right| \\
& \geqslant\left(1-\frac{\delta}{3}\right)\left|\alpha_{n}\right| \cdot\left|u_{n-1}\left(\mathbf{f}_{1}\right)\right| \geqslant \cdots \geqslant\left(1-\frac{\delta}{3}\right)^{n-n_{1}}\left|\alpha_{n} \cdots \alpha_{n-n_{1}+1}\right| \cdot\left|u_{n_{1}}\left(\mathbf{f}_{1}\right)\right| \\
& =\left(1-\frac{\delta}{3}\right)^{n-n_{1}}\left|\alpha_{n} \cdots \alpha_{n-n_{1}+1}\right| .
\end{aligned}
$$

Покажем теперь, что найдется вектор $\mathbf{f}_{2}$, для которого $\left|u_{n}\left(\mathbf{f}_{2}\right) / v_{n}\left(\mathbf{f}_{2}\right)\right| \leqslant c^{1 / 2} \varepsilon^{-1 / 2}$ при всех $n \geqslant n_{1}$. Выберем из последовательности векторов

$$
\mathbf{f}^{k}=\mathbf{A}_{1}^{-1} \cdots \mathbf{A}_{k}^{-1} \mathbf{e}_{2}\left\|\mathbf{A}_{1}^{-1} \cdots \mathbf{A}_{k}^{-1} \mathbf{e}_{2}\right\|^{-1}
$$

сходящуюся подпоследовательность

$$
\lim _{l \rightarrow \infty} \mathbf{f}^{k_{l}}=\mathbf{f}_{2} .
$$

Предположим, что $\left|u_{n}\left(\mathbf{f}_{2}\right) / v_{n}\left(\mathbf{f}_{2}\right)\right|>c^{1 / 2} \varepsilon^{-1 / 2}$ при некотором $n=n_{2} \geqslant n_{1}$. Возьмем вектор $\mathbf{f}^{k_{l}}\left(k_{l}>n_{2}\right)$ настолько близким к $\mathbf{f}_{2}$, чтобы и $\left|u_{n_{2}}\left(\mathbf{f}^{k_{l}}\right) / v_{n_{2}}\left(\mathbf{f}^{k_{l}}\right)\right|>c^{1 / 2} \varepsilon^{-1 / 2}$. Но тогда по доказанному $\left|u_{n}\left(\mathbf{f}^{k_{l}}\right) / v_{n}\left(\mathbf{f}^{k_{l}}\right)\right| \geqslant c^{1 / 2} \varepsilon^{-1 / 2}$ при всех $n \geqslant n_{2}$ и, в частности, при $n=k_{l}$, что невозможно, так как по построению

$$
\begin{aligned}
u_{k_{l}}\left(\mathbf{f}^{k_{l}}\right) & =\left[\mathbf{A}_{k_{l}} \cdots \mathbf{A}_{1}\left(\mathbf{A}_{1}^{-1} \cdots \mathbf{A}_{k_{l}}^{-1} \mathbf{e}_{2}\right)\right]^{1}\left\|\mathbf{A}_{1}^{-1} \cdots \mathbf{A}_{k_{l}}^{-1} \mathbf{e}_{2}\right\|^{-1} \\
& =\left[\mathbf{e}_{2}\right]^{1}\left\|\mathbf{A}_{1}^{-1} \cdots \mathbf{A}_{k_{l}}^{-1} \mathbf{e}_{2}\right\|^{-1}=0, \quad v_{k_{l}}\left(\mathbf{f}^{k_{l}}\right) \neq 0 .
\end{aligned}
$$

Полученное противоречие показьвает, что при всех $n \geqslant n_{1}$ будет вьполнено $\left|u_{n}\left(\mathbf{f}_{2}\right) / v_{n}\left(\mathbf{f}_{2}\right)\right| \leqslant c^{1 / 2} \varepsilon^{-1 / 2}$ и, следовательно,

$$
\begin{aligned}
\left|v_{n}\left(\mathbf{f}_{2}\right)\right| & =\left|\gamma_{n} u_{n-1}\left(\mathbf{f}_{2}\right)+\delta_{n} v_{n-1}\left(\mathbf{f}_{2}\right)\right| \leqslant\left(\varepsilon\left(c^{1 / 2} \varepsilon^{-1 / 2}\right)+(1-\delta)\right)\left|\alpha_{n}\right| \cdot\left|v_{n-1}\left(\mathbf{f}_{2}\right)\right| \\
& \leqslant\left(1-\frac{2 \delta}{3}\right)\left|\alpha_{n}\right| \cdot\left|v_{n-1}\left(\mathbf{f}_{2}\right)\right| \leqslant \cdots \\
& \leqslant\left(1-\frac{2 \delta}{3}\right)^{n-n_{1}}\left|\alpha_{n} \cdots \alpha_{n-n_{1}+1}\right| \cdot\left|v_{n_{1}-1}\left(\mathbf{f}_{2}\right)\right|, \\
\left|u_{n}\left(\mathbf{f}_{2}\right)\right| & \leqslant c^{1 / 2} \varepsilon^{-1 / 2}\left|v_{n}\left(\mathbf{f}_{2}\right)\right| \leqslant c^{1 / 2} \varepsilon^{-1 / 2}\left(1-\frac{2 \delta}{3}\right)^{n-n_{1}}\left|\alpha_{n} \cdots \alpha_{n-n_{1}+1}\right| \cdot\left|v_{n_{1}-1}\left(\mathbf{f}_{2}\right)\right| .
\end{aligned}
$$

Сравнивая последние два неравенства с (9), получаем (8).

Докажем теперь (7), т.е. докажем, что

$$
\lim _{n \rightarrow \infty} \frac{v_{n}\left(\mathbf{f}_{1}\right)}{u_{n}\left(\mathbf{f}_{1}\right)}=0 .
$$

Для любого сколь угодно малого $\tau>0$ при всех $n \geqslant n(\tau)$ имеем

$$
\begin{aligned}
\left|\frac{v_{n}\left(\mathbf{f}_{1}\right)}{u_{n}\left(\mathbf{f}_{1}\right)}\right| & =\left|\frac{\gamma_{n} u_{n-1}\left(\mathbf{f}_{1}\right)+\delta_{n} v_{n-1}\left(\mathbf{f}_{1}\right)}{\alpha_{n} u_{n-1}\left(\mathbf{f}_{1}\right)+\beta_{n} v_{n-1}\left(\mathbf{f}_{1}\right)}\right| \leqslant \frac{\tau\left|u_{n-1}\left(\mathbf{f}_{1}\right)\right|+(1-\delta)\left|v_{n-1}\left(\mathbf{f}_{1}\right)\right|}{\left|u_{n-1}\left(\mathbf{f}_{1}\right)\right|-c\left(\varepsilon^{1 / 2} c^{-1 / 2}\right)\left|u_{n-1}\left(\mathbf{f}_{1}\right)\right|} \\
& \leqslant \frac{\tau+(1-\delta)\left|v_{n-1}\left(\mathbf{f}_{1}\right) / u_{n-1}\left(\mathbf{f}_{1}\right)\right|}{1-\delta / 3} .
\end{aligned}
$$


Обозначая через $\Lambda$ подпоследовательность натуральных чисел, по которой достигается верхний предел

$$
\varlimsup_{n \rightarrow \infty}\left|\frac{v_{n}\left(\mathbf{f}_{1}\right)}{u_{n}\left(\mathbf{f}_{1}\right)}\right|=\rho,
$$

из последнего неравенства имеем

$\rho \geqslant \varlimsup_{n \in \Lambda}\left|\frac{v_{n-1}\left(\mathbf{f}_{1}\right)}{u_{n-1}\left(\mathbf{f}_{1}\right)}\right| \geqslant \varlimsup_{n \in \Lambda}\left[\left(1-\frac{\delta}{3}\right)\left|\frac{v_{n}\left(\mathbf{f}_{1}\right)}{u_{n}\left(\mathbf{f}_{1}\right)}\right|-\tau\right](1-\delta)^{-1}=\left[\left(1-\frac{\delta}{3}\right) \rho-\tau\right](1-\delta)^{-1}$.

Ввиду произвольной малости $\tau$ это неравенство возможно лиш при $\rho=0$. Таким обpaзом,

$$
0=\varlimsup_{n \rightarrow \infty}\left|\frac{v_{n}\left(\mathbf{f}_{1}\right)}{u_{n}\left(\mathbf{f}_{1}\right)}\right|=\lim _{n \rightarrow \infty}\left|\frac{v_{n}\left(\mathbf{f}_{1}\right)}{u_{n}\left(\mathbf{f}_{1}\right)}\right|,
$$

т.е. равенство (7) доказано.

Из равенств (7) и (8) следует также и линейная независимость векторов $\mathbf{f}_{1}$ и $\mathbf{f}_{2}$. Лемма 1 доказана.

ЛЕмма 2. Пусть в двумерном векторном пространстве имеется последовательность невырожсденных линейных операторов $\mathbf{A}_{n}(n=1,2, \ldots)$, задаваемых в некотором фиксированном базисе $\mathbf{e}_{1}, \mathbf{e}_{2}$ равенствами $\mathbf{A}_{n} \mathbf{e}_{1}=\alpha_{n} \mathbf{e}_{1}+\gamma_{n} \mathbf{e}_{2}, \mathbf{A}_{n} \mathbf{e}_{2}=$ $\beta_{n} \mathbf{e}_{1}+\delta_{n} \mathbf{e}_{2}$, где $\alpha_{n} \neq 0\left(n \geqslant n_{0}\right)$,

$$
\lim _{n \rightarrow \infty} \beta_{n} \alpha_{n}^{-1}=0, \quad \varlimsup_{n \rightarrow \infty}\left|\gamma_{n} \alpha_{n}^{-1}\right|<\infty, \quad \varlimsup_{n \rightarrow \infty}\left|\delta_{n} \alpha_{n}^{-1}\right|<1 .
$$

Тогда для всякого вектора $\mathbf{f}$ вида $\mathbf{f}=\mathbf{e}_{1}+$ бе $\mathbf{e}_{2}$ последовательность векторов $d_{n} \mathbf{A}_{1} \cdots \mathbf{A}_{n} \mathbf{f}(n=1,2, \ldots)$, где $d_{n}$ - некоторая фиксированная числовая последовательность, имеет ненулевой предел, и этот предел не зависит от $\xi$.

ДокАЗАТЕЛЬСТво. В сопряженном векторном пространстве линейных функционалов введем базис $\mathbf{e}_{1}^{*}, \mathbf{e}_{2}^{*}$, сопряженньй исходному, т.е. $\left\langle\mathbf{e}_{i}, \mathbf{e}_{j}^{*}\right\rangle=\delta_{i j}$, где $\delta_{i j}-$ символ Кронекера. Тогда для сопряженных операторов $\mathbf{A}_{n}^{*}(n=1,2, \ldots)$ имеем $\mathbf{A}_{n}^{*} \mathbf{e}_{1}^{*}=\alpha_{n} \mathbf{e}_{1}^{*}+\beta_{n} \mathbf{e}_{2}^{*}$, $\mathbf{A}_{n}^{*} \mathbf{e}_{2}^{*}=\gamma_{n} \mathbf{e}_{1}^{*}+\delta_{n} \mathbf{e}_{2}^{*}$, и, следовательно, последовательность операторов $\mathbf{A}_{n}^{*}(n=1,2, \ldots)$ удовлетворяет всем условиям леммы 1 . Поэтому в сопряженном пространственайдутся два вектора $\mathbf{g}_{1}^{*}$ и $\mathbf{g}_{2}^{*}$ такие, что

$$
\lim _{n \rightarrow \infty} d_{n} \mathbf{A}_{n}^{*} \cdots \mathbf{A}_{1}^{*} \mathbf{g}_{1}^{*}=\mathbf{e}_{1}^{*}, \quad \lim _{n \rightarrow \infty} d_{n} \mathbf{A}_{n}^{*} \cdots \mathbf{A}_{1}^{*} \mathbf{g}_{2}^{*}=0
$$

где $d_{n}=\left(\left[\mathbf{A}_{n}^{*} \cdots \mathbf{A}_{1}^{*} \mathbf{g}_{1}^{*}\right]^{1}\right)^{-1}$. Тогда для всякого $\mathbf{g}^{*}=\zeta_{1} \mathbf{g}_{1}^{*}+\zeta_{2} \mathbf{g}_{2}^{*}$

$$
\begin{aligned}
\lim _{n \rightarrow \infty}\left\langle d_{n} \mathbf{A}_{1} \cdots \mathbf{A}_{n} \mathbf{f}, \mathbf{g}^{*}\right\rangle & =\lim _{n \rightarrow \infty} d_{n}\left\langle\mathbf{f}, \mathbf{A}_{n}^{*} \cdots \mathbf{A}_{1}^{*} \mathbf{g}^{*}\right\rangle=\left\langle\mathbf{f}, \zeta_{1} \mathbf{e}_{1}^{*}\right\rangle=\left\langle\mathbf{e}_{1}+\xi \mathbf{e}_{2}, \zeta_{1} \mathbf{e}_{1}^{*}\right\rangle \\
& =\left\langle\mathbf{e}_{1}, \zeta_{1} \mathbf{e}_{1}^{*}\right\rangle=\left\langle\mathbf{e}_{1}, \zeta_{1} \mathbf{e}_{1}^{*}+\zeta_{2} \mathbf{e}_{2}^{*}\right\rangle=\left\langle\mathbf{e}_{1}, \mathbf{T}^{*} \mathbf{g}^{*}\right\rangle=\left\langle\mathbf{T} \mathbf{e}_{1}, \mathbf{g}^{*}\right\rangle,
\end{aligned}
$$

где $\mathbf{T}^{*}$ - линейньй оператор, переводяший $\mathbf{g}_{1}^{*}$ в $\mathbf{e}_{1}^{*}, \mathbf{g}_{2}^{*}$ в $\mathbf{e}_{2}^{*}$. Так как данное равенство справедливо для любого сопряженного вектора $\mathbf{g}^{*}$, то

$$
\lim _{n \rightarrow \infty} d_{n} \mathbf{A}_{1} \cdots \mathbf{A}_{n} \mathbf{f}
$$

существует и равен $\mathbf{T e}_{1}$. Лемма 2 доказана. 
ДоКАЗАТЕЛЬСТВо ТЕОРЕМЫ 2. Не нарушая обшности рассуждений, можно считать, что $p_{2}=\infty$. В противном случае этого можно добиться переходом к последовательности дробно-линейных преобразований $\mathbf{T}_{n}(z)=\left(\mathbf{Q}^{-1} \circ \mathbf{S}_{n} \circ \mathbf{Q}\right)(z)(n=1,2, \ldots)$, где $\mathbf{Q}$ - некоторое фиксированное дробно-линейное преобразование такое, что $\mathbf{Q}(\infty)=p_{2}$.

Пусть $\mathbf{S}_{n}(z)=\left(\alpha_{n} z+\beta_{n}\right) /\left(\gamma_{n} z+\delta_{n}\right)$. Рассмотрим последовательность линейных операторов $\mathbf{A}_{n}(n=1,2, \ldots)$, задаваемых в некотором фиксированном базисе $\mathbf{e}_{1}, \mathbf{e}_{2}$ равенствами $\mathbf{A}_{n} \mathbf{e}_{1}=\alpha_{n} \mathbf{e}_{1}+\gamma_{n} \mathbf{e}_{2}, \mathbf{A}_{n} \mathbf{e}_{2}=\beta_{n} \mathbf{e}_{1}+\delta_{n} \mathbf{e}_{2}$, и покажем, что эта последовательность операторов удовлетворяет всем условиям леммы 1. Из равенств

$$
\begin{aligned}
& \gamma_{n}=\frac{\alpha_{n}-\delta_{n}}{p_{2, n}}+\frac{\beta_{n}}{p_{2, n}^{2}} \\
& \beta_{n}=\left(\delta_{n}-\alpha_{n}\right) p_{1, n}+\gamma_{n} p_{1, n}^{2}
\end{aligned}
$$

и условий $(1),(2)$ теоремы 2 следует, что

$$
\gamma_{n}=o\left(m_{n}\right), \quad \beta_{n}=O\left(m_{n}\right), \quad \text { где } m_{n}=\max \left\{\left|\alpha_{n}\right|,\left|\delta_{n}\right|\right\} .
$$

С учетом этих оценок на $\gamma_{n}, \beta_{n}$, условия (3) теоремы 2 и равенства $\mathbf{S}^{\prime}\left(p_{1, n}\right) \mathbf{S}^{\prime}\left(p_{2, n}\right)=1$ имеем

$$
\begin{aligned}
1>\varlimsup_{n \rightarrow \infty}\left|\mathbf{S}_{n}^{\prime}\left(p_{2, n}\right)\right|=\varlimsup_{n \rightarrow \infty}\left|\mathbf{S}_{n}^{\prime}\left(p_{1, n}\right)\right|^{-1} & =\varlimsup_{n \rightarrow \infty} \frac{\left|\gamma_{n} p_{1, n}+\delta_{n}\right|^{2}}{\left|\alpha_{n} \delta_{n}-\gamma_{n} \beta_{n}\right|} \\
& =\varlimsup_{n \rightarrow \infty} \frac{\left|\delta_{n}^{2}\right|+o\left(m_{n}^{2}\right)}{\left|\alpha_{n} \delta_{n}\right|+o\left(m_{n}^{2}\right)} .
\end{aligned}
$$

Отсюда следует, что

$$
\varlimsup_{n \rightarrow \infty}\left|\delta_{n} \alpha_{n}^{-1}\right|<1 .
$$

Полученные оценки показьвают, что для последовательности операторов $\mathbf{A}_{n}(n=1$, $2, \ldots)$ вьполнены все условия леммы 1.

Заметим, что $\left(\mathbf{S}_{n} \circ \cdots \circ \mathbf{S}_{1}\right)(z)=\left[\mathbf{A}_{n} \cdots \mathbf{A}_{1} \mathbf{z}\right]^{1}\left(\left[\mathbf{A}_{n} \cdots \mathbf{A}_{1} \mathbf{z}\right]^{2}\right)^{-1}$, где $\mathbf{z}-$ двумерньй вектор с координатами $(z, 1)$ (при $z=\infty \mathbf{z}=(0,1))$. Пусть $\mathbf{f}_{1}$ и $\mathbf{f}_{2}-$ два двумерньх вектора, для которых справедливы предельные равенства (7) и (8) из леммы 1. Тогда для всех $z \neq z_{0}$, где $z_{0}=\left[\mathbf{f}_{2}\right]^{1}\left(\left[\mathbf{f}_{2}\right]^{2}\right)^{-1}, \mathbf{z}$ представляется в виде $\mathbf{z}=\xi_{1} \mathbf{f}_{1}+\xi_{2} \mathbf{f}_{2}\left(\xi_{1} \neq 0\right)$ и по лемме 1

$$
\begin{aligned}
\lim _{n \rightarrow \infty}\left(\mathbf{S}_{n} \circ \cdots \circ \mathbf{S}_{1}\right)(z) & =\lim _{n \rightarrow \infty}\left[D_{n}^{-1} \mathbf{A}_{n} \cdots \mathbf{A}_{1} \mathbf{z}\right]^{1}\left(\left[D_{n}^{-1} \mathbf{A}_{n} \cdots \mathbf{A}_{1} \mathbf{z}\right]^{2}\right)^{-1} \\
& =\left[\xi_{1} \mathbf{e}_{1}\right]^{1}\left(\left[\xi_{1} \mathbf{e}_{1}\right]^{2}\right)^{-1}=\infty
\end{aligned}
$$

Теорема 2 доказана.

ДоКАЗАТЕЛЬСТВо ТЕОРЕМЫ 1. Не нарушая обшности рассуждений, можно считать, что $p_{1}=0$. Рассмотрим последовательность операторов $\mathbf{A}_{n}(n=1,2, \ldots)$, определенных точно так же, как и в доказательстве теоремы 2 . Из равенств (10), (11) и условий теоремы 1 теперь следует, что $\beta_{n}=o\left(m_{n}\right), \gamma_{n}=O\left(m_{n}\right)$ и

$$
\varlimsup_{n \rightarrow \infty}\left|\delta_{n} \alpha_{n}^{-1}\right|<1 \text {, }
$$

т.е. для последовательности операторов $\mathbf{A}_{n}(n=1,2, \ldots)$ выполнены все условия леммы 2. Таким образом, при всех $z \neq p_{1}$, т.е. при всех $\mathbf{z} \neq \mathbf{e}_{2}$

$$
\lim _{n \rightarrow \infty}\left(\mathbf{S}_{1} \circ \cdots \circ \mathbf{S}_{n}\right)(z)=\lim _{n \rightarrow \infty}\left[d_{n} \mathbf{A}_{1} \cdots \mathbf{A}_{n} \mathbf{z}\right]^{1}\left(\left[d_{n} \mathbf{A}_{1} \cdots \mathbf{A}_{n} \mathbf{z}\right]^{2}\right)^{-1}
$$

и последний предел сушествует по лемме 2. Теорема 1 доказана. 
ДОКАЗАТЕЛЬСТВО ТЕОРЕМЫ 3. Как уже отмечалось, последовательность подходяших дробей непрерывной дроби (4) совпадает с последовательностью композиций $\left(\mathbf{S}_{1} \circ \cdots \circ \mathbf{S}_{n}\right)(0)(n=1,2, \ldots)$ дробно-линейных преобразований $\mathbf{S}_{n}(z)=\alpha_{n} /\left(\beta_{n}+z\right)$. Поэтому достаточно показать, что для этой последовательности дробно-линейных преобразований условия теоремы 1 являются следствиями условий теоремы 3.

Неподвижные точки этих дробно-линейных преобразований вычисляются по формуле $p_{n}=\left(-\beta_{n} \pm\left(\beta_{n}^{2}+4 \alpha_{n}\right)^{1 / 2}\right) 2^{-1}$. Подставляя в эту формулу выражение для $\beta_{n}$, найденное из (6): $\beta_{n}=\alpha_{n} p^{-1}-p+o(1)$, и учитьвая, что $|p|>\left|\alpha_{n} p^{-1}\right|\left(n \geqslant n_{0}\right)$, имеем

$$
p_{n}=\left(p-\alpha_{n} p^{-1} \pm\left(p+\alpha_{n} p^{-1}\right)\right) 2^{-1}+o(1),
$$

т.е. $p_{1, n}=p+o(1), p_{2, n}=-\alpha_{n} p^{-1}+o(1)$. Таким образом,

$$
\lim _{n \rightarrow \infty} p_{1, n}=p, \quad\left|p-p_{2, n}\right| \geqslant|p|-\left|\alpha_{n} p^{-1}+o(1)\right|>0 \quad\left(n \geqslant n_{1}\right),
$$

а с учетом неравенств (5) справедливо также и неравенство

$$
\varliminf_{n \rightarrow \infty}\left|\mathbf{S}_{n}^{\prime}\left(p_{1, n}\right)\right|=\varliminf_{n \rightarrow \infty}\left|\frac{\alpha_{n}}{\left(\beta_{n}+p_{1, n}\right)^{2}}\right|=\lim _{n \rightarrow \infty} \frac{\left|\alpha_{n}\right|}{\left|\alpha_{n} p^{-1}+o(1)\right|^{2}}=\varliminf_{n \rightarrow \infty}\left|\frac{p^{2}}{\alpha_{n}}\right|>1,
$$

т.е. все предположения теоремы 1 вьполнены. Теорема 3 доказана.

Математический институт им. В. А. Стеклова РАН

Поступило

Институт математики НАН Украины

10.11 .96

\section{СПИСОК ЦИТИРОВАННОЙ ЛИТЕРАТУРЫ}

[1] Van Vleck E. B. On the convergence of algebraic continued fractions whose coefficients have limiting values // Trans. Amer. Math. Soc. 1904. V. 5. P. 253-262.

[2] Poincaré H. Sur les équations linéaires aux différentielles et aux différences finies // Amer. J. Math. 1885. V. 7. P. 203-258.

[3] Перрон О. Об одной теореме А. Пуанкаре // J. Reine Angew. Math. 1909. V. 136. P. 17-37; О линейном разностном уравнении А. Пуанкаре // J. Reine Angew. Math. 1910. V. 137. P. 6-64.

[4] Гельфонд А. О. Исчисление конечных разностей. М.: Наука, 1967.

[5] Фрейман Г. А. О теоремах Пуанкаре и Перрона // УМН. 1957. Т. 12. № 3. С. 241-246.

[6] Maté A., Nevai P. A generalization of Poincaré's theorem for recurrence equations // J. Approx. Theory. 1990. V. 63. P. 92-97. 